\title{
A bird pollinator shows positive frequency dependence and constancy of species choice in natural plant communities
}

\author{
B. Schmid,${ }^{1,2,6,7}$ H. Nottebrock, ${ }^{2,3,4}$ K. J. Esler, ${ }^{2}$ J. Pagel,${ }^{3,4}$ K. Böhning-Gaese, ${ }^{1,5}$ F. M. Schurr, ${ }^{3,4}$ \\ T. Mueller, ${ }^{1,5}$ AND M. Schleuning ${ }^{1}$ \\ ${ }^{1}$ Senckenberg Biodiversity and Climate Research Centre (BiK-F), Senckenberganlage 25, Frankfurt am Main, 60325 Germany \\ ${ }^{2}$ Department of Conservation Biology and Entomology and Centre for Invasion Biology, Stellenbosch University, Private Bag X1, \\ Matieland 7602 South Africa \\ ${ }^{3}$ Institute of Landscape and Plant Ecology, University of Hohenheim, August-von-Hartmann-Street 3, Stuttgart 70599 Germany \\ ${ }^{4}$ Institut des Sciences de l'Evolution, UMR 5554, Université Montpellier 2, Place Eugène Bataillon, Montpellier Cedex 05 \\ F-34095 France \\ ${ }^{5}$ Department of Biological Sciences, Johann Wolfgang Goethe-University, Max-von-Laue-Straße 9, Frankfurt am Main \\ 60438 Germany \\ ${ }^{6}$ Swiss Ornithological Institute 6204 Sempach, Switzerland
}

\begin{abstract}
Animal pollinators mediate reproduction of many plant species. Foraging theory suggests that animal pollinators exhibit preferences for common plant species in natural communities (positive frequency-dependent foraging) and temporary single-species specialization (flower constancy) during foraging bouts. Positive frequency dependence may favor common plant species; flower constancy may enhance conspecific pollen transfer particularly in rare plant species. Previous experimental studies suggest that avian pollinators are unlikely to exhibit these behaviors. We studied foraging behavior of Cape Sugarbirds (Promerops cafer), the main avian pollinator of many Protea species, using focal-plant and focal-bird sampling, assisted by high-resolution maps of the spatiotemporal distribution of Protea individuals and their flowering status. We found that Sugarbird's visitation preference increased with species' relative floral abundance, and that individual Sugarbirds tended to visit single species in sequence. Flower constancy during foraging bouts was significantly higher than expected from random plant-animal encounters at the scale of pollinator movements. Positive frequency dependence may favor the reproduction of abundant plant species while flower constancy may be particularly important for rare plant species. This first simultaneous study of both behaviors in a natural plant-pollinator system shows that bird pollinators exhibit both types of behavior and, in this way, possibly influence plant community structure.
\end{abstract}

Key words: animal movement; Cape Floristic Region; Cape Sugarbird; flower constancy; foraging preference; frequency-dependent foraging; pollination; Proteaceae; step selection functions.

\section{INTRODUCTION}

Animal pollinators forage on plant resources optimizing their net energy intake (Stephens and Kerbs 1986) while simultaneously providing essential services to plant species in ecological communities (Waser and Ollerton 2006). According to optimal foraging theory (MacArthur and Pianka 1966), foraging choices of animal pollinators change according to the distributions of plant resources and the local context (Bateson et al. 2003, Hersch and Roy 2007). This context dependence in the foraging behavior of animal pollinators can influence plant reproduction (Rathcke 1983, Brosi and Briggs 2013) and thus the dynamics of plant communities (Kunin and Iwasa 1996, Song and Feldman 2014).

Two mechanisms of context-dependent foraging behavior that ought to facilitate conspecific pollen transfer have been proposed, positive frequency-dependent

Manuscript received 1 March 2016; revised 4 June 2016; accepted 21 July 2016. Corresponding Editor: E. H. DuVal.

${ }^{7}$ E-mail: baptiste.schmid@vogelwarte.ch foraging and flower constancy (Waser 1986, Smithson 2001). Positive frequency dependence of pollinators occurs when a pollinator visits common plant species at higher frequency than expected from a random choice among co-occurring plant species (Krebs 1989, Fründ et al. 2010). Flower constancy describes the foraging behavior of individual pollinators that continue to forage on the same species, bypassing other rewarding species (Waser 1986). Flower constancy thus describes a temporary specialization of pollinator individuals (Chittka et al. 1999), a foraging behavior that differs from the widespread traplining behavior of pollinators along regular foraging routes (Thomson et al. 1997). One possible mechanism generating these two foraging behaviors is the cognitive limitation of pollinators to efficiently forage on multiple flower types simultaneously (Chittka et al. 1999, Gegear and Laverty 2001, Menzel 2001, but see Grüter and Ratnieks 2011). For instance, foraging efficiency improves when pollinators activate search images to accelerate and refine the detection of suitable flowers (Goulson 2000, Smithson 2001). Controlled experimental 
studies demonstrate that animal pollinators show both positive frequency-dependent foraging (Levin 1972, reviewed in Smithson 2001) and flower constancy (Chittka et al. 1999). However, studies in controlled environments are difficult with avian pollinators and the investigation of foraging constancy under natural conditions is very difficult due to the heterogeneous distribution of plant resources. Previous studies suggest that avian pollinators show no or weak flower constancy in experimental plant arrays (Waser 1978, Meléndez-Ackerman et al. 1997, Hersch and Roy 2007), maybe because avian pollinators repeatedly assess resource availability in novel experimental environments (Meléndez-Ackerman et al. 1997).

Positive frequency dependence and flower constancy of animal pollinators have rarely been studied in natural, species-rich, plant communities (de Jager et al. 2011) and, to our knowledge, they have never been studied simultaneously. Evidence for frequency-dependent foraging from field studies is ambiguous (discussed in Smithson 2001, see also Eckhart et al. 2006), and evidence for flower constancy is rare because it is difficult to account for the non-random, heterogeneous distributions of plants at spatial scales corresponding to pollinator movements (Waser 1986, de Jager et al. 2011). Here we investigate the foraging behavior of Cape Sugarbirds (Promerops cafer), a key pollinator for Protea species in the Cape Floristic Region (Collins and Rebelo 1987, Schmid et al. 2015a). We mapped all plants of two to seven co-flowering Protea species at 14 4-ha study plots in natural plant communities (Fig. 1A) and tested frequency-dependent foraging of Sugarbirds with observations of foraging events on focal plants (Fig. 1B). We additionally determined foraging constancy by recording Sugarbird movements between foraging events (Fig. 1B) and applying step-selection functions to compare observed with random encounter probabilities between pollinators and plants (Fig. 1C, see Thurfjell et al. 2014).

We hypothesized that (1) Cape Sugarbirds adjust their foraging choices in response to the relative abundance of flowering plants, preferring common over rare species, as observed in other pollinators (Levin 1972, Smithson 2001), and that (2) Cape Sugarbirds show no foraging constancy during subsequent foraging events, like observed in other bird pollinators (Waser 1978, MeléndezAckerman et al. 1997, Hersch and Roy 2007). Because male and female Sugarbirds differ in morphology and behavior (Seiler and Rebelo 1987), we also explored (3) whether the two sexes differ in frequency dependence and flower constancy.

\section{Methods}

\section{Study system}

We investigated the foraging plasticity of a generalist avian pollinator, the Cape Sugarbird (Promerops cafer, Family Promeropidae). During the breeding season, Cape Sugarbirds depend on nectar resources provided almost exclusively by a large variety of Protea species (Rebelo 1987) and are considered key pollinators for many of these species (Collins and Rebelo 1987, Schmid et al. 2015a). Sugarbirds' morphology and behavior differ between sexes. Males are territorial and have larger bodies and longer beaks than females (Seiler and Rebelo 1987, Tjørve and Scholtz 2007).

We studied interactions between Sugarbirds and Protea species in the Cape Floristic Region (CFR; Goldblatt 1978) in the Western Cape, South Africa. To this end, we selected 27 study plots where Protea species dominated the overstorey vegetation (Appendix S1: Fig.

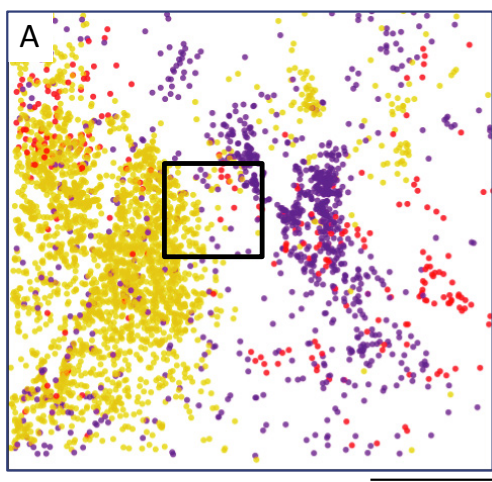

$50 \mathrm{~m}$

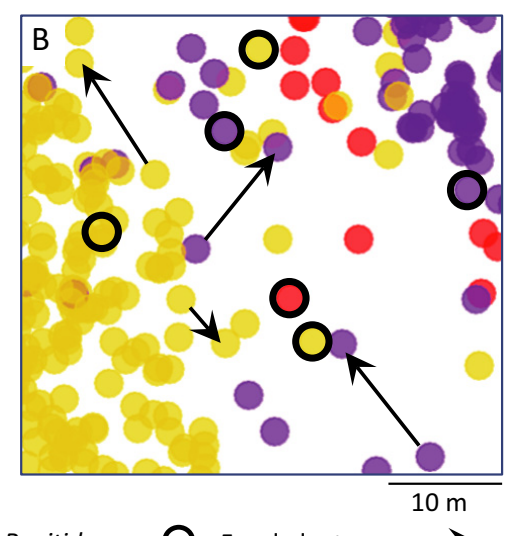

P. nitida

Focal plants

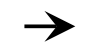

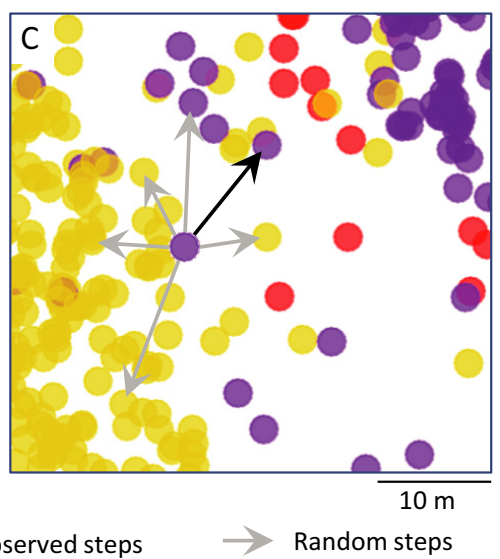

FIG. 1. Observations of foraging Cape Sugarbirds in Protea communities. (A) Example for the mapped spatial distribution of individual plants of three Protea species (study plot 11 in Appendix S1: Fig. S1); the square indicates the area displayed in panels B and C. (B) Example of randomly selected focal plants (black circles) used to study frequency-dependent foraging and observed bird movements between consecutive foraging events (black arrows) used to investigate foraging constancy. (C) For each observed movement step (black arrow), we sampled 50 random steps (gray arrows) that share the same start-plant, but can differ in endplants. End-plants of random steps are selected at distances that correspond to the lengths of the observed movement steps (Appendix S2: Fig. S1) and thus account for the spatially heterogeneous distribution of flowering plants. 
S1). We defined a $200 \times 200 \mathrm{~m}$ plot larger than the size of individual Sugarbird territories (Calf et al. 2003). We mapped all overstorey Protea plants with a high accuracy Differential Global Positioning System (Trimble GeoXH, Sunnyvale, California, USA) and recorded the size of each plant (stem length along the main growth axis). The study plots varied in the number of co-flowering Protea species (two to seven species) and overall plant abundance $(650-15,500$ plants). Only study plots providing high abundances of floral Protea resources hosted Sugarbirds (Schmid et al. 2015b). We thus restricted the observation of the foraging activity of Sugarbirds to the 14 study plots where Sugarbirds occupied permanent territories (Appendix S1: Fig. S1).

We observed bird foraging activity during the breeding season of Sugarbirds and flowering peak of Protea species from April to August 2012 and repeated observations every $2-5$ weeks for a total of three visits per study plot. During each visit, we recorded (1) the number of Sugarbird visits to focal plants and (2) the movements of foraging Sugarbirds within the study plot. We restricted our analyses to the 10 Protea species for which we recorded foraging events while following Sugarbirds movements: $P$. coronata ( $n=24$ visits), $P$. cynaroides $(n=11)$, P. eximia $(n=15)$, $P$. laurifolia $(n=42), P$. longifolia $(n=38), P$. neriifolia $(n=53), P$. nitida $(n=5)$, P. obtusifolia $(n=41), P$. repens $(n=101)$, and $P$. susannae $(n=60)$.

\section{Spatiotemporal distribution of flowering plants}

Since repeated counts of all flowering Protea plants would have been impossible across the 14 plots, we estimated the spatiotemporal distribution of flowering Protea plants in the study plots for each day of observation by combining two statistical models of inflorescence production with the spatial mapping data. First, we estimated the flowering phenology for each Protea species. To this end, we recorded information on the flowering status (i.e., the estimated number of flowering individuals) for 15,863 populations (48-4145 populations per Protea species) provided by the Protea Atlas Project (Rebelo 2001). Second, we counted the number of inflorescences on focal plants in the study plots $(n=6,943$ observations, $51-1,245$ per species, $1-865$ per plot). On the same plants, we also recorded plant size, inflorescence length, trunk length, and specific leaf area. Since the survival probability after bushfires (sprouting vs. nonsprouting Protea species) influences species' reproductive strategy and the number of inflorescences produced per year, we also recorded information on the sprouting ability of each species (Rebelo 2001).

We then combined these different sources of information in two statistical models to predict the number of inflorescences for each mapped Protea individual on the days of observation. First, we estimated the phenological peak of flower production for each Protea species from the Protea Atlas data (i.e., we determined the day in the year when most plant individuals of a specific Protea species were flowering). Second, we related the number of inflorescences of each mapped plant on a given day to the temporal distance from the day of the flowering peak (as derived from the first model), individual plant size, and a set of plant functional traits (all plant traits are listed in Appendix S1: Fig. S2), using a generalized linear mixed model (GLMM) with Poisson errors. We used the estimates of this model to predict the number of inflorescences for each mapped plant and day of observation. This yielded spatially explicit maps of flowering plants for each plot at the days of bird observation. The data sources and statistical models used for predicting the spatiotemporal distribution of flowering Protea plants are summarized in Appendix S1: Fig. S2 (see also Schmid et al. 2015b).

\section{Frequency-dependent foraging}

We assessed frequency-dependent foraging of male and female Sugarbirds on each study plot. To this end, we defined the relative abundance of plant species from the pollinator perspective as the mean relative abundance of flowering Protea species on each plot. To calculate the proportion of flowering plants for each Protea species on each observation day, we used the predicted spatiotemporal distribution of flowering plants on each plot. For the analysis of frequency-dependent foraging, we computed the mean relative floral abundance of each Protea species over the three days of bird observations for each plot (see Appendix S1: Table S1 for abundance estimates per plot and species).

To assess the foraging behavior of Sugarbirds, we observed 1,122 focal plants (12-358 focal plants per Protea species, one to eight study plots per species, depending on the availability of flowering plants; Fig. 1A). Focal plants were selected according to species' relative abundances, i.e., frequently flowering plant species were observed more frequently than rare species. We observed focal plants from a distance of at least $20 \mathrm{~m}$ during 45 -min sessions in the morning from 07:00 to 12:00. We considered only bird visits to focal plants with legitimate feeding events, i.e., birds that were in contact with the reproductive parts of an inflorescence and potentially transferred pollen. Results of analyses including legitimate and illegitimate bird visits were qualitatively identical. As proposed by Fründ et al. (2010) and based on Krebs' forage ratio (Krebs 1989), we calculated relative preference indices for each species $s$ on each study plot $p$, pooling the observations from the three repeated visits per plot. The preference index $\left(\mathrm{PI}_{s, p}\right)$ ranges between 0 and 1, and was calculated for each Sugarbird sex as

$$
\mathrm{PI}_{s, p}=P_{\mathrm{obs} s, p} /\left(P_{\mathrm{obs} s, p}+P_{\text {null } s, p}\right)
$$

where $P_{\text {obs s.p }}$ is the proportion of visits on species $s$ among all visits on plot $p$, and $P_{\text {null } s, p}$ is the proportion of focal plants of species $s$ among all focal plants observed 
on plot $p$. We thus control for the number of focal plants observed for each Protea species. $\mathrm{PI}_{s, p}$ is zero if no interactions with species $s$ were observed on study plot $p ; \mathrm{PI}_{s, p}$ equals 0.5 when the observed frequency of interactions matches the expectation according to the observation effort for species $s$. PI approaches 1 for strong preferences of pollinators, but will never be much larger than 0.5 for frequently observed plant species, i.e., large values of $P_{\text {null } s, p}$.

\section{Flower constancy}

We conducted direct observations of movement paths of unmarked bird individuals and used bird movements between consecutive foraging events to examine flower constancy. For the examination of flower constancy, we compared the observed proportion of consecutive foraging events on two conspecific plants with the respective proportions expected from random pollinator movements (Gegear and Laverty 2005, de Jager et al. 2011). In contrast to former studies on flower constancy (Gegear and Laverty 2005, de Jager et al. 2011), we explicitly integrated pollinator movements and the heterogeneous distribution of plants to compute random encounter rates with plant species (Fig. 1C). This approach resembles case-control designs in studies of habitat selection of moving animals (Fortin et al. 2005, Thurfjell et al. 2014). We define a step as the Euclidian distance between consecutive foraging events, geographically defined by the location of a foraging event (start-plant) and the consecutive foraging event (end-plant). If the bird continued to fly to other plants and foraged more than twice, we considered all consecutive steps to belong to the same foraging bout; the identity of the foraging bout was included as a random factor in the GLMM (see details in the data analyses section). We coupled each case step (i.e., observed foraging step; $n=257$ ) with 50 random steps that shared the same start-plant, but could differ in endplants. We chose 50 random steps to obtain a representative random sample of potential plants within the foraging range of individual birds, given the long computing times for these simulations. Random end-plants were selected from the predicted spatial distribution of flowering plants (see Appendix S2: Fig. S1) and thus the selection of end-plants accounted for the spatially heterogeneous distribution of flowering plants. We drew case step distances (with replacement) to select the random end-plants matching the observed distance between startand end-plant (Appendix S2: Fig. S1; Fortin et al. 2005). It is possible that the detection probability of foraging events decreases with increasing distance of the bird from the observer. However, density distributions of distances from the observer to case end-plants were similar to those to random end-plants and thus we did not find any evidence for biases, due to changes in detection probability (Appendix S2: Fig. S1). Because we were also interested in sex differences in foraging choice, we drew step distances for each sex separately.

\section{Data analyses}

Positive frequency-dependent foraging.-We tested the relationship between preference indices (range, 0-1; angular-transformed) per species and plot and (1) relative plant species abundance and (2) sex of Sugarbirds, assuming Gaussian error distributions. Because bird sexes might differ in their foraging preferences, we also included the two-way interaction of bird species and relative species abundance. We included random intercept effects of plot identity and Protea species identity to account for potential spatial and taxonomic autocorrelation in preference indices. To ensure comparability among predictor variables, all numerical predictors were scaled and centered. We applied Wald's $\chi^{2}$ tests to assess the level of significance for each predictor variable; each predictor variable was tested with all other variables in the statistical model, accounting for their respective effects. We used mixed effects models as implemented in the lme4 package (version 1.0-6; Bates et al. 2014) of $\mathrm{R}$ (version 3.1.0; R Core Team 2014).

Foraging constancy.-We used mixed logistic regression models to test whether the probability of conspecific foraging sequences in observed movement steps differed from the probability of conspecific foraging sequences in random steps. The response variable was a binary variable that indicated for each foraging step whether the startplant and the end-plant belonged to the same species. We included the relative species abundance of end-plants as predictor variable. For each case step and its coupled random steps, we computed the relative species abundance of end-plants in the 50 random steps (sampling procedure of random steps described in subsection Flower constancy), as derived from the predictions of flowering Protea plants for each plot and observation day. We further included as predictors the categorical variables step mode (observed step or random step) and bird sex (male or female). The main effect of relative species abundance of end-plants accounted for encounter probabilities that are due to the relative frequency of end plants; the main effect of step mode (observed vs. random step) indicated whether the foraging choices of birds deviated from the random expectation given by the relative species abundance of end-plants. To explore whether foraging constancy of observed birds might be influenced by bird sex, we also included bird sex as main and interaction effects with step mode in the model. A significant interaction effect of sex with step mode would indicate whether the observed probabilities of foraging constancy differed between sexes. In all models of foraging constancy, we also included random intercepts of the species identity of end-plants to account for between species differences, and nested random intercepts of step identity, movement path identity, date, and plot identity to account for spatial and temporal autocorrelation. We repeated the sampling and analyses 1,000 times to estimate the range of parameter values. To ensure comparability among predictor variables, all numerical 
TABLE 1. Effects of relative species abundance and sex of Sugarbirds on avian foraging preferences.

\begin{tabular}{lccc}
\hline \hline Effects & Model estimate $\pm \mathrm{SE}$ & $\chi^{2}$ & $P$ \\
\hline $\begin{array}{l}\text { Relative species } \\
\quad \text { abundance }\end{array}$ & $\mathbf{0 . 1 4 0} \pm \mathbf{0 . 0 6 5}$ & $\mathbf{1 2 . 0 0}$ & $<\mathbf{0 . 0 0 1}$ \\
Sex (female) & $-0.058 \pm 0.080$ & 0.52 & 0.47 \\
$\begin{array}{l}\text { Relative species } \\
\quad \text { abundance } \times \text { sex }\end{array}$ & $0.030 \pm 0.080$ & 0.14 & 0.70 \\
\hline
\end{tabular}

Notes: Preference indices (angular transformed; mean $\pm \mathrm{SE}$ ) are given by the relative number of bird visits to focal plants of a specific species among all focal plants on a study plot. All numerical predictors were scaled and centered. Significant effects $(P \leq 0.001)$ according to Wald's $\chi^{2}$ tests are shown in boldface type (marginal model $R^{2}=0.25$ ). The model accounts for random effects (in parentheses) of plot identity (variance $\pm \mathrm{SD}$, $0.014 \pm 0.119)$ and species identity $(0.019 \pm 0.138)$ on preference indices.

predictors were scaled and centered. We used mixed effects models as implemented in the lme 4 package (version 1.0-6; Bates et al. 2014) of R (version 3.1.0; R Core Team 2014). We did not use two-step conditional logistic models ( $\mathrm{R}$ package TwoStepCLogit, version 1.2.3; Craiu et al. 2014) because it does not support crossed random effects, such as species identity, plot identity, and date.

\section{Results}

\section{Positive frequency-dependent foraging}

Cape Sugarbirds of both sexes showed positive frequency-dependent foraging. For 10 Protea species, we observed 190 bird visits (female Sugarbirds, $n=69$; male Sugarbirds, $n=121$ ) during 115 h of observation on 711 focal plants. Preference indices ranged between 0 and 0.84 and increased with the relative abundance of flowering plants of the respective species (Table 1, Fig. 2). Preference indices were independent of pollinator sex and the two-way interaction between species abundance and bird sex (Table 1).

\section{Flower constancy}

Observed movements between consecutive foraging events were, in most cases, directed to conspecific plants ( $79 \%, n=257$ observations). The odds that birds showed flower constancy were 2.39 (median $\log$-odds $=0.87$; parameter range after $10^{3}$ permutations, 0.71-1.07) times greater in the observed movement steps than expected from the random encounter frequencies (Fig. 3). Importantly, the effect size of flower constancy, i.e., the ratio between observed and random encounter probabilities as predicted by the statistical model, was higher for rare than for common plant species (relative species abundance $\leq 0.20$, effect size $\geq 2.75$; relative species abundance $\geq 0.75$, effect size $\approx 1$; see Fig. 3A). Consequently, the probability to forage on a conspecific plant relative to a random encounter was particularly large at a low relative species abundance (Fig. 3B).

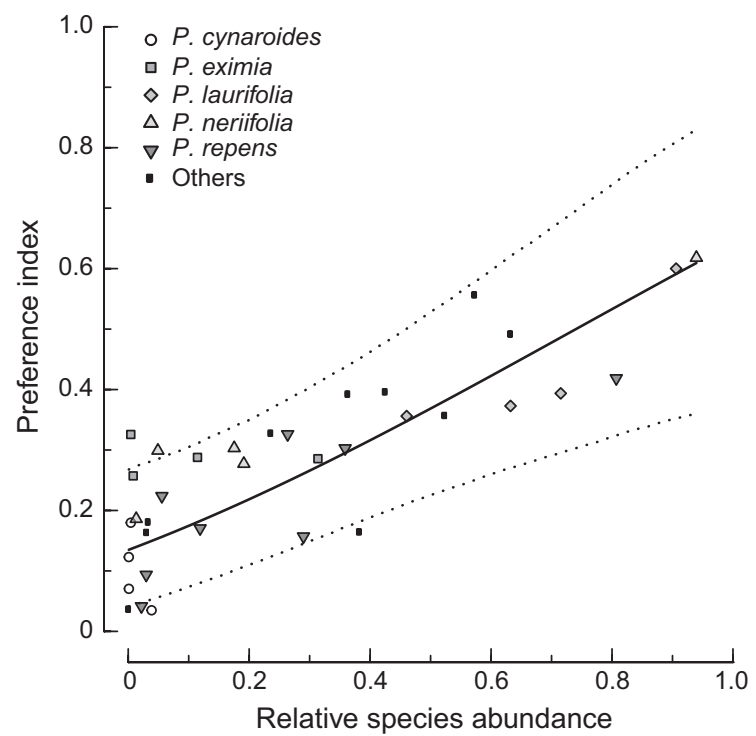

FIG. 2. Relationship between indices of Sugarbird foraging preferences for 10 Protea species and the relative abundances of flowering plants of the respective species on 14 study plots. Protea species include (number of study plots in parentheses) $P$. cynaroides (4), P. eximia (4), P. laurifolia (4), P. neriifolia (5), $P$. repens, (8) and others (with less than four study plots), including $P$. coronata $(2), P$. longifolia $(2), P$. nitida (1), $P$. obtusifolia (3), and $P$. susannae (2). We computed posterior values (points) and predicted values of fixed effects (solid line; dotted lines represent the $0.025 / 0.975$ confidence intervals) using models that included only relative species abundance as fixed effect, since bird sex showed no significant effects (intercept $0.53 \pm 0.07$ [mean $\pm \mathrm{SE}$ ], relative species abundance $0.16 \pm 0.05$; species identity is included as random intercept; marginal model $\left.R^{2}=0.24\right)$.

As expected, increasing relative species abundance increased the probability that birds continued to forage on the same species simply because the encounter probability of common species was higher than that of rare species (median log-odds $=2.98$; parameter range 2.75 3.25; Fig. 3). We observed no differences in flower constancy between bird sexes (observed movements of females contrasted against the observed movements of males: median log-odds $=0.31$; parameter range -0.05 $0.71)$. The effects of relative species abundance on foraging probabilities deviate from linearity because of the effects of the random intercepts included in the statistical model; variance and standard deviation of random effects (median across 1000 permutations; species identity $=14.63 \pm 4.64$, plot identity $=10.80 \pm 3.29$, date $=1.19 \pm 1.08$, path identity $=2.88 \pm 1.70$, step identity $=2.97 \pm 1.72$ ).

\section{Discussion}

We studied the context-dependent foraging behavior of an avian pollinator in natural species-rich plant communities. We provide first evidence for flower constancy of avian pollinators under natural conditions and 
A

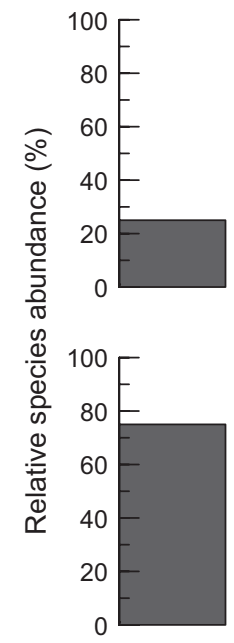

i) Without constancy (null model)

\section{ii)}

With constancy (observed)

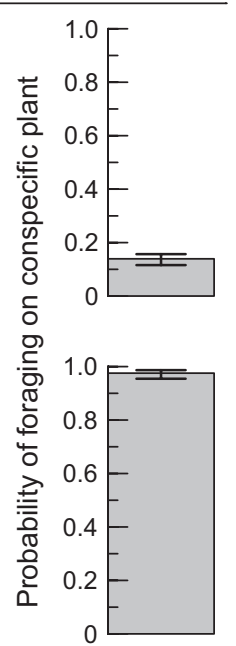

iii) Effect size of constancy

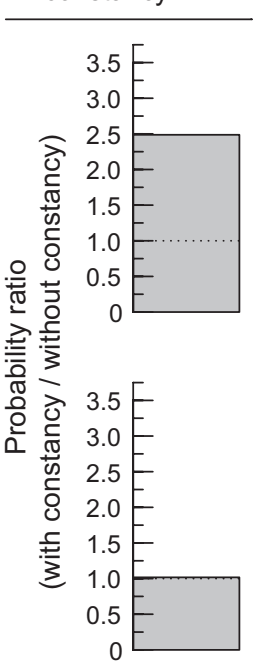

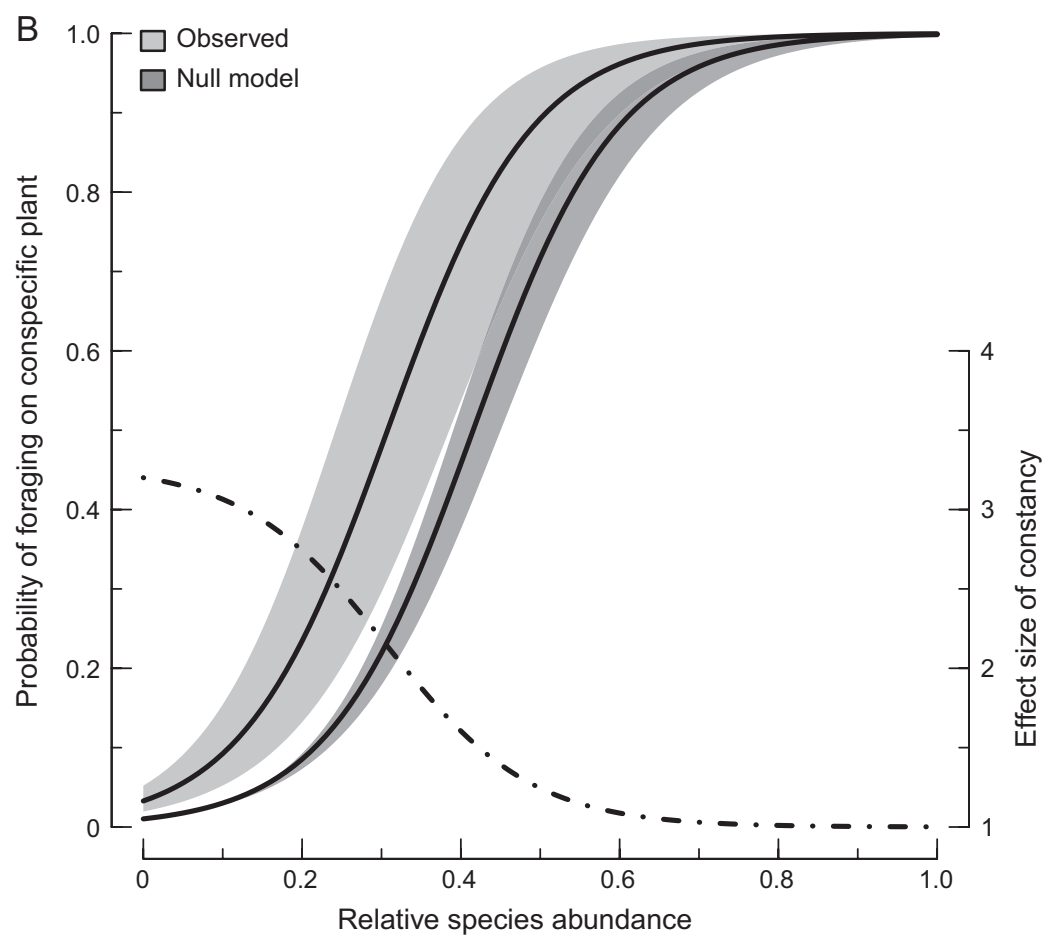

FIG. 3. Flower constancy of Cape Sugarbirds on 14 study plots. (A) The predicted probability of foraging on a conspecific plant for a rare and an abundant plant species: (i) according to random steps, and (ii) according to the observed bird movements (case steps). The ratio of observed to random probabilities indicates (iii) the effect size of flower constancy for a given species abundance. We used the median and the $0.025 / 0.975$ intervals of the parameter values of the 1000 models to compute predicted means and error bars. (B) The predicted probability of foraging on a conspecific plant (left axis) in relation to the relative abundance of plant species according to random steps (null model, dark gray), and according to the observed bird movements (observed, light gray). Shaded areas correspond to the $0.025 / 0.975$ confidence intervals of the relationship according to the model parameters. We also show how the ratio of the observed probabilities relative to the random probabilities (i.e., the effect size of flower constancy) changes with increasing relative species abundance (right axis; dashed line).

additional evidence for positive frequency-dependent foraging of avian pollinators in species-rich plant communities. Morphological and behavioral differences among bird sexes did not influence foraging choices.
Positive frequency dependence favors the reproduction of locally common plant species while flower constancy may be particularly important for rare species, promoting conspecific pollen transfer. The prevalence of foraging 
constancy may be particularly important in asymmetric plant-pollinator interaction systems, where generalist pollinators forage on many co-flowering plant species. Our results thus highlight the importance of animal foraging behavior for plant-animal interactions.

\section{Positive frequency-dependent foraging}

Cape Sugarbirds, regardless of their sex, preferred to forage on common plant species. According to optimal foraging theory (MacArthur and Pianka 1966), foraging on common species may optimize the foraging efficiency of animals (Smithson 2001). Positive frequency-dependent foraging, for instance, occurs when pollinators develop search images for common species (Levin 1972, Chittka and Thomson 1997). The search-image hypothesis has received much support in predator-prey interactions (e.g., Dawkins 1971), but the importance of search images in plant-pollinator interactions is contentious, due to contradictory findings of foraging studies under field conditions (reviewed in Smithson 2001, see also Eckhart et al. 2006). Smithson (2001) suggested that the discrepancy in the incidence of frequency-dependent foraging between experimental and field studies might be explained by the comparatively small floral polymorphism of plant species under natural conditions. Large interspecific variation in floral traits among Protea species (Rebelo 2001), e.g., in the size, shape, and flower of inflorescences, might therefore be an important factor promoting frequencydependent foraging in the Protea-Sugarbird system.

\section{Flower constancy}

Cape Sugarbirds prefer to continue foraging on the same species, bypassing other rewarding plant species. We detected this floral constancy of Sugarbirds in models that also accounted for frequency-dependent foraging. Our study is the first that explicitly considers the spatially heterogeneous distribution of resource plants at a spatial resolution directly related to the range of pollinator movements. Our approach generates accurate random encounter frequencies with co-occurring species (Waser 1986) and thus allows testing floral constancy of animal pollinators in natural species-rich plant communities.

We further detect an important difference in the effect size of flower constancy between rare and common plant species. We found that the probability of visiting conspecific plants increased strongly for rare plants (Fig. 3B) that benefitted most from the flower constancy of Sugarbirds. In contrast, in the case of abundant plants, Sugarbirds tended to forage on the same plant species irrespective of flower constancy (Fig. 3B). The small effect size of flower constancy for abundant species can be explained by the higher encounter probabilities for abundant plant species and may be independent from positive frequency-dependent foraging.

The significant flower constancy of Sugarbirds is surprising since pollinators with high cognitive abilities, such as birds (Henderson et al. 2006), are expected to be able to identify different resources and are therefore expected to show low incidences of flower constancy (Meléndez-Ackerman et al. 1997). However, the low incidence of flower constancy of avian pollinators in experimental arrays may be mostly driven by their need to explore the resource availability of different resource types, as suggested by Meléndez-Ackerman et al. (1997). In contrast, feral avian pollinators may gather long-term experience in resource availability and may therefore be able to accurately assess changes in resource availability (Henderson et al. 2006) without excessive explorative foraging. Flower constancy of Sugarbirds in natural plant communities can be explained by several, non-mutually exclusive explanations. First, limitations in processing simultaneous information have been observed also in organisms with high cognitive abilities, such as humans (discussed in Goulson 2000). Avian pollinators may improve foraging efficiency by the use of search images, especially in species-rich plant communities with large variation in floral traits (Gegear and Laverty 2001, 2005). Thus, variation in floral traits among Protea species (Rebelo 2001) may promote flower constancy of Sugarbirds. Second, plant species may differ in the timing of nectar production rates within a day and pollinators may thus specialize temporarily on the most rewarding species. Information on the interspecific variability in nectar production rates among Protea species is lacking. However, flowers in Protea inflorescences open sequentially over the course of several days to weeks (Rebelo 2001), and Protea species differ in the daily pattern of flower opening within inflorescences (Wiens et al. 1983). Therefore, it is likely that Protea species also differ in the daily pattern of nectar production rates. Third, differences in the competitive ability and energy requirements of individual pollinators within a population can lead to individualistic foraging choices and high degrees of foraging specialization (Thomson and Chittka 2001, Brosi and Briggs 2013). Breeding Sugarbirds might indeed show strong individual differences since males intensively interact with conspecific males during courtship and territorial defense (Seiler and Rebelo 1987). Further studies with individually marked bird individuals would be required to thoroughly disentangle the underlying mechanisms of flower constancy in avian pollinators.

\section{Potential effects of foraging behavior on plant reproduction}

The plasticity in the foraging behavior of Cape Sugarbirds may strongly influence plant reproduction in Protea communities. Positive frequency-dependent foraging can increase the reproductive success of common plant species to the detriment of rare species (Palmer et al. 2003). Consequently, rare species may experience reduced fitness because of decreased conspecific pollen transfer (Thomson and Plowright 1980, Rathcke 1983, Flanagan et al. 2009). However, the local extinction risk 
of rare plant species might be reduced by pollinator behavior in two ways. First, pollinator species that differ in competitive ability may show distinct frequencydependent foraging preferences, with subordinate pollinators showing preferences for rare species (Eckhart et al. 2006). For instance, Orange-breasted Sunbirds (Anthobaphes violacea) also regularly forage on Protea species, but are often chased away from Protea inflorescences by dominant Cape Sugarbirds (Wooller 1982, Rebelo 1987). Thus, subordinate Orange-breasted Sunbirds may prefer rare plant species unattended by Sugarbirds. Second, the flower constancy of individual Sugarbirds may improve the efficiency of conspecific pollen carry-over and mitigate indirect competition for pollinators between plants. Flower constancy may thus be particularly important to enhance the reproduction of rare plant species.

\section{Conclusion}

Avian pollination is important in many tropical and sub-tropical ecosystems (Stiles 1981, Rebelo 1987). While interactions between plants and Hummingbirds are usually rather specialized (Dalsgaard et al. 2011), other avian pollinators such as Honeyeaters, Sunbirds, and Sugarbirds are less specialized and often exploit floral resources of many different plant species (Rebelo 1987, Fleming and Muchhala 2008). In such asymmetric and generalized plant-pollinator systems, conspecific pollen transfer may crucially depend on the temporary specialization of pollinator individuals on specific plant resources. Here we show that Sugarbirds indeed exhibit floral constancy in highly diverse plant communities and thereby increase conspecific pollen transfer especially for rare plant species. This finding is the first evidence for floral constancy of avian pollinators in natural species-rich plant communities. It is likely that the individual foraging behavior of pollinators is an important, and thus far largely neglected, mechanism for the coexistence between common and rare plant species, especially in ecosystems where many plant species depend on a few keystone pollinators.

\section{ACKNOWLEDGMENTS}

We are thankful for assistance and advice to P. Barnard, T. Caprano, C. Devaux, M. Forrest, S. Gallegos, R. O'Hara, A. Pauw, T. Rebelo, and M. Templin. We warmly thank L. Ingmann, M. Nowell, B. Olivier, S. Rossow, and P. Strauss for their commitment during field work. We were able to accomplish our study thank to the friendly collaboration of private land owners (Floraleae Farm, Paarl; Flower Valley, Gansbaai; Grootbos Nature Reserve, Gansbaai; Heidehof, Gansbaai; Heuningklip Farm, Kleinmond; High Noon Farm, Villiersdorp; G. Moskovitz and S. Moskovitz, DuToitskloof pass) and nature reserve managers and their collaborators (Fernkloof Nature Reserve, Helderberg Nature Reserve, Hottentots-Holland Nature Reserve, Jonaskop Nature Reserve, Limietsberg Nature Reserve, Mont-Rochelle Nature Reserve). We also thank the reviewers for their valuable comments on the manuscript. Field work was conducted under the CapeNature permit
AAA005-00213-0028. This work was supported by the German Research Foundation [grant numbers SCHL 1934/1-1, SCHU 2259/3-1] and by the research funding programme LOEWELandes-Offensive zur Entwicklung Wissenschaftlich-ökonomischer Exzellenz of Hesse's Ministry of Higher Education, Research, and the Arts. Thomas Müller was supported by the Robert Bosch Foundation.

\section{Literature Cited}

Bates, D., M. Maechler, B. Bolker, and S. Walker. 2014. lme4: linear mixed-effects models using Eigen and S4.

Bateson, M., S. D. Healy, and T. A. Hurly. 2003. Contextdependent foraging decisions in rufous hummingbirds. Proceedings of the Royal Society B 270:1271-1276.

Brosi, B. J., and H. M. Briggs. 2013. Single pollinator species losses reduce floral fidelity and plant reproductive function. Proceedings of the National Academy of Sciences USA 110:13044-13048.

Calf, K., C. Downs, and M. Cherry. 2003. Territoriality of Cape Sugarbirds (Promerops cafer) between and within breeding seasons. Ostrich 74:125-128.

Chittka, L., and J. D. Thomson. 1997. Sensori-motor learning and its relevance for task specialization in bumble bees. Behavioral Ecology and Sociobiology 41:385-398.

Chittka, L., J. D. Thomson, and N. M. Waser. 1999. Flower constancy, insect psychology, and plant evolution. Naturwissenschaften 86:361-377.

Collins, B. G., and A. G. Rebelo. 1987. Pollination biology of the Proteaceae in Australia and southern Africa. Austral Ecology 12:387-421.

Craiu, R. V., T. Duchesne, D. Fortin, and S. Baillargeon. 2014. TwoStepCLogit: conditional logistic regression: a two-step estimation method. R Foundation for Statistical Computing. http://cran.r-project.org/package=TwoStepCLogit

Dalsgaard, B., et al. 2011. Specialization in plant-hummingbird networks is associated with species richness, contemporary precipitation and quaternary climate-change velocity. PLoS ONE 6:e25891.

Dawkins, M. 1971. Perceptual changes in chicks: another look at the "search image" concept. Animal Behaviour 19:566574 .

de Jager, M. L., L. L. Dreyer, and A. G. Ellis. 2011. Do pollinators influence the assembly of flower colours within plant communities? Oecologia 166:543-553.

Eckhart, V., N. Rushing, G. Hart, and J. Hansen. 2006. Frequency-dependent pollinator foraging in polymorphic Clarkia xantiana ssp. xantiana populations: implications for flower colour evolution and pollinator interactions. Oikos $112: 412-421$

Flanagan, R. J., R. J. Mitchell, D. Knutowski, and J. D. Karron. 2009. Interspecific pollinator movements reduce pollen deposition and seed production in Mimulus ringens (Phrymaceae). American Journal of Botany 96:809-815.

Fleming, T. H., and N. Muchhala. 2008. Nectar-feeding bird and bat niches in two worlds: pantropical comparisons of vertebrate pollination systems. Journal of Biogeography 35: 764-780.

Fortin, D., H. L. Beyer, M. S. Boyce, D. W. Smith, T. Duchesne, and J. S. Mao. 2005. Wolves influence elk movements: behavior shape a trophic cascade in Yellowstone National Park. Ecology 86:1320-1330.

Fründ, J., K. E. Linsenmair, and N. Blüthgen. 2010. Pollinator diversity and specialization in relation to flower diversity. Oikos 119:1581-1590.

Gegear, R. J., and T. M. Laverty. 2001. The effect of variation among floral traits on the flower constancy of pollinators. 
Pages 1-20 in L. Chittka and J. D. Thomson, editors. Cognitive ecology of pollination. Cambridge University Press, Cambridge, UK.

Gegear, R., and T. Laverty. 2005. Flower constancy in bumblebees: a test of the trait variability hypothesis. Animal Behaviour 69:939-949.

Goldblatt, P. 1978. An analysis of the flora of southern Africa: its characteristics, relationships, and origins. Annals of the Missouri Botanical Garden 65:369-436.

Goulson, D. 2000. Are insects flower constant because they use search images to find flowers? Oikos 88:547-552.

Grüter, C., and F. Ratnieks. 2011. Flower constancy in insect pollinators: adaptive foraging behaviour or cognitive limitation? Communicative \& Integrative Biology 4:633-636.

Henderson, J., T. A. Hurly, M. Bateson, and S. D. Healy. 2006. Timing in free-living rufous hummingbirds, Selasphorus rufus. Current Biology 16:512-515.

Hersch, E. I., and B. A. Roy. 2007. Context-dependent pollinator behavior: an explanation for patterns of hybridization among three species of Indian paintbrush. Evolution 61:111-124.

Krebs, C. J. 1989. Ecological methodology. Harper \& Row, New York, New York, USA.

Kunin, W., and Y. Iwasa. 1996. Pollinator foraging strategies in mixed floral arrays: density effects and floral constancy. Theoretical Population Biology 49:232-263.

Levin, D. 1972. Low frequency disadvantage in the exploitation of pollinators by corolla variants in Phlox. American Naturalist 106:453-460.

MacArthur, R. H., and E. R. Pianka. 1966. On optimal use of a patchy environment. American Naturalist 100:603-609.

Meléndez-Ackerman, E., D. R. Campbell, and N. M. Waser. 1997. Hummingbird behavior and mechanisms of selection on flower color in Ipomopsis. Ecology 78:2532-2541.

Menzel, R. 2001. Behavioral and neural mechanisms of learning and memory as determinants of flower constancy. Pages 3344 in L. Chittka and J. D. Thomson, editors. Cognitive ecology of pollination. Cambridge University Press, Cambridge, UK.

Palmer, T. M., M. L. Stanton, and T. P. Young. 2003. Competition and coexistence: exploring mechanisms that restrict and maintain diversity within mutualist guilds. American Naturalist 162:S63-S79.

R Core Team. 2014. R: a language and environment for statistical computing. R Foundation for Statistical Computing, Vienna, Austria.

Rathcke, B. 1983. Competition and facilitation among plants for pollination. Pages 305-329 in L. Real, editor. Pollination biology. Academic Press, New York, New York, USA.

Rebelo, A. G. 1987. Bird pollination in the Cape Flora. Pages 83-108 in A. G. Rebelo, editor. Preliminary synthesis of pollination biology in the Cape Flora. CSIR, Pretoria, South Africa.

Rebelo, A. G. 2001. Proteas: a field guide to the Proteas of Southern Africa. Pages 0-240 in E. Du Plessis, editor. Fernwood Press, Cape Town, South Africa.
Schmid, B., H. Nottebrock, K. J. Esler, J. Pagel, A. Pauw, K. Böhning-Gaese, F. M. Schurr, and M. Schleuning. 2015a. Reward quality predicts effects of bird-pollinators on the reproduction of African Protea shrubs. Perspectives in Plant Ecology, Evolution and Systematics 17:209-217.

Schmid, B., H. Nottebrock, K. J. Esler, J. Pagel, A. Pauw, K. Böhning-Gaese, F. M. Schurr, and M. Schleuning. $2015 b$. Responses of nectar-feeding birds to floral resources at multiple spatial scales. Ecography 38:001-011.

Seiler, H. W., and A. G. Rebelo. 1987. A sexual difference in the Cape Sugarbird's role as pollinator of Protea lepidocarpodendron. Ostrich 58:43-45.

Smithson, A. 2001. Pollinator preference, frequency dependence, and floral evolution. Pages 237-259 in L. Chittka and J. D. Thomson, editors. Cognitive ecology of pollination. Cambridge University Press, Cambridge, UK.

Song, Z., and M. W. Feldman. 2014. Adaptive foraging behaviour of individual pollinators and the coexistence of co-flowering plants. Proceedings of the Royal Society B 281:20132437.

Stephens, D. W., and J. R. Kerbs. 1986. Foraging theory. Princeton University Press, Princeton, New Jersey, USA.

Stiles, F. G. 1981. Geographical aspects of bird-flower coevolution, with particular reference to Central America. Annals of the Missouri Botanical Garden 68:323-351.

Thomson, J. D., and L. Chittka. 2001. Pollinator individuality: when does it matter. Pages 191-213 in L. Chittka and J. D. Thomson, editors. Cognitive ecology of pollination. Cambridge University Press, Cambridge, UK

Thomson, J. D. D., and R. C. C. Plowright. 1980. Pollen carryover, nectar rewards, and pollinator behavior with special reference to Diervilla lonicera. Oecologia 74:68-74.

Thomson, J., M. Slatkin, and B. Thomson. 1997. Trapline foraging by bumble bees: II. Definition and detection from sequence data. Behavioral Ecology 8:199-210.

Thurfjell, H., S. Ciuti, and M. S. Boyce. 2014. Applications of step-selection functions in ecology and conservation. Movement Ecology 2:4.

Tjørve, K. M. C., and G. Scholtz. 2007. Morphological characteristics of Cape sugarbirds (Promerops cafer) from Helderberg Nature Reserve. African Zoology 42:199-203.

Waser, N. M. 1978. Competition for hummingbird pollination and sequential flowering in two Colorado wildflowers. Ecology 59:934-944.

Waser, N. M. 1986. Flower constancy: definition, cause, and measurement. American Naturalist 127:593-603.

Waser, N. M., and J. Ollerton. 2006. Plant-pollinator interactions, from specialization to generalization. University of Chicago Press, Chicago, Illinois, USA.

Wiens, D., J. P. Rourke, and B. B. Casper. 1983. Nonflying mammal pollination of southern African Proteas: a noncoevolved system. Annals of the Missouri Botanical Garden 70:1-31.

Wooller, R. D. 1982. Feeding interactions between sunbirds and sugarbirds. Ostrich 53:114-115.

\section{SUPPORTING INFORMATION}

Additional supporting information may be found in the online version of this article at http://onlinelibrary.wiley.com/ doi/10.1002/ecy.1565/suppinfo

\section{Data Availability}

The data used in this study are archived in the Senckenberg repository: http://dataportal-senckenberg.de/database/metacat/ bikf.373.1/bikf. 